\title{
Design and Application of A New Hybrid Heuristic Algorithm for Flow Shop Scheduling
}

\author{
Fang Wang and Yun-qing Rao \\ Huazhong University of Science and Technology/State Key Lab of Digital Manufacturing Equipment and Technology, \\ Wuhan, PR China \\ wfang305@163.com and ryq@mail.hust.edu.cn \\ Fang Wang and $\mathrm{Yu}$ Hou \\ Wuhan University of Science and Technology/School of management, Wuhan, PR China \\ wfang305@163.com and fly_houyu@163.com
}

\begin{abstract}
-a new heuristic algorithm was designed by combining with Johnson method, NEH method and characteristics of scheduling, and it was implemented on MATLAB. The efficiency of the new algorithm was tested through eight Car questions and two Hel questions of Benchmark problems, and the results revealed that the new heuristic algorithm was better than the other three heuristic algorithms. Further more; the application of this heuristic algorithm in the intelligent algorithm especially in the genetic algorithms (GA) was discussed. Two GAs were designed for Flow Shop question, and they had the same processes and the same parameters. The only difference is in the production of the initial population. One GA's initial population is optimized by the new heuristic algorithm, and the other whose initial population is randomly generated entirely. Finally, through the test of eight Car questions, it is demonstrated that the heuristic algorithm can indeed improve efficiency and quality of genetic algorithm because the heuristic algorithm can improve the initial population of GA.
\end{abstract}

Index Terms-heuristic algorithm; genetic algorithm; Benchmark problems test; initial population

\section{INTRODUCTION}

Flow Shop scheduling problem can be described as that it is $\mathrm{n}$ work pieces go through the m machines with the same techniques, and one of its main objectives is to find out the work pieces arrangement which makes the maximum completion time to be shortest. In the Flow Shop Scheduling, there are mixed integer linear programming, branch and bound Etc. exact algorithms. Because Flow Shop problem is a part of NP-Complete problem, these methods can not be calculated on the large-scale problems [1]. Besides the exact techniques which are applicable only to small problems in practice, currently available methods for the flow shop problem may be classified as constructive heuristic algorithm and intelligent search algorithm. In K. R. Bake' book of "sequencing and scheduling" we can find detailed description about Gupta algorithm, Johnson, Palmer and CDS heuristics algorithms [2], and in the article which had been written by M. Nawaz, E. Enscore Jr and I. Ham in 1983 proposed NEH method [3], in 1998 Koulamos proposed a new heuristic algorithm which had the same performance with NEH [4]. In 2003 Wang Ling summarizes the various heuristic methods, and noted that $\mathrm{NEH}$ and Rajendran approaches may be the most effective practice of polynomial heuristics [5]. With the rapid development of computer technology, the improved methods, such as simulated annealing (SA) [6], genetic algorithm (GA) [7], and tabu search (TS) [8], have gained much attention during the last decade for overcoming the non-flexibility of the constructive methods. Genetic algorithm is one kind of evolutionary computation which is created by Professor John. H. Holland [9] who is from the University of Michigan, the book "Adaption in Natural and Artificial System" (Holland, 1975) marked the Birth of the genetic algorithm. The monograph "Genetic Algorithms in Search, Optimization and Machine Learning" (Goldberg, 1989) written by David. E. Dr. Goldberg [10], gave a comprehensive overview of the development process and present situation of genetic algorithms, and gave a variety of algorithms and examples together with Pascal source code to allow the engineers and technicians to carry out the actual application. But the intelligent algorithm has slow convergence speed and is easy to fall into local optimal solution, and it must refer to heuristic methods to get better results. It can be said, the quality of constructive heuristic methods directly determines the performance of intelligent methods. Therefore, L. Wang and D. Z. Zheng proposed a hybrid algorithm based on the NEH and the GA[11], and CEYDA OG UZ \& M. FIKRET ERCAN also proposed a improved genetic algorithm for HFS problem[12]. In this paper, based on the characteristics of work pieces, NEH and Dannenbring methods, a new constructive heuristic algorithm is proposed, and it is implemented based on MATLAB. A genetic algorithm for flow shop is designed, and after two experimental groups is designed, the initial population of one group is optimized by the new heuristic algorithm, and the initial population of the other is randomly generated entirely, the improvement efficiency of this heuristic algorithm is tested through eight Car questions.

\section{II . INTRODUCTIONS OF RELATIONAL ALGORITHMS}




\section{A. Johnson Method}

Johnson method is mainly used for scheduling two machines. First, the minimum time of processing the work pieces on the machine has been find out, if the time is in the first machine, then the processing sequence of this work piece is the first; if the time is in the second machine, then the processing sequence of this work piece is the last. Second, the rest work pieces are arranged in the same way until there is no work piece to wait for arrangement.

\section{B. Dannenbring Method}

Dannenbring method is the expansion of Johnson method, it extends the Johnson method to more than two machines scheduling problem. Through the following two formulas (formula 1 and formula 2 ), the multi-machine scheduling problem converses into the two-machine scheduling problem, and the suboptimal solution can be obtained by Johnson method.

$$
\begin{gathered}
T_{j 1}=\sum_{i=1}^{m}(m-i+1) * t_{i j} . \\
T_{j 2}=\sum_{i=1}^{m} i^{*} t_{i j} .
\end{gathered}
$$

\section{NEH Method}

First, calculate the sum of processing time of each work piece on all machines, and order each work piece according to the descending order of the sum. Second conduct the optimal scheduling on the first two work piece, and insert one of the rest work pieces into the scheduling to obtain the optimal schedule, repeat this process until all the work pieces scheduling is completed.

\section{Rajendran Method}

Rajendran is to modify the methods of the NEH. First, get the $\mathrm{Tj} 1$ after handling the processing time by formula 1 , and arrange the work pieces scheduling by the ascending order of $\mathrm{Tj} 1$. Second, contrive a subscheduling about the first work piece; take No. $\mathrm{k}$ work piece into No. L positions of the scheduling, where [k/2] $<=\mathrm{L}<=\mathrm{k}, \mathrm{k}>=2$, and make the optimal scheduling for the new sub-schedule. The last, repeat this process until all the work pieces scheduling is completed.

In these heuristic algorithms, NEH method and Rajendren method has the best performance. But there are always more than one optimal schedule in each cycle, when the number of the work pieces is big, computational complexity will rapidly increase. Such as when finding the optimal solution of Hell problem by $\mathrm{NEH}$, if saving the optimal sequences every time, the number of optimal Scheduling will be more than 100,000 for No.40 work piece. With the number of work pieces addition, it will exceed the scope of the computer's memory. Therefore, this paper proposes a new hybrid constructive heuristic algorithm based on the complexity of the number of work pieces.

\section{E. Genetic Algorithm}

A genetic algorithm (GA) is a search technique used in computing to find exact or approximate solutions to optimization and search problems. Genetic algorithms are categorized as global search heuristics. Genetic algorithms are a particular class of evolutionary algorithms (EA) that use techniques inspired by evolutionary biology such as inheritance, mutation, selection, and crossover.

\section{The NEW Hybrid CONSTRUCTIVE HeURISTIC ALGORITHM}

\section{A. Idea of Flow Shop Problem Solving with Hybrid Constructive Heuristic Algorithm}

According to the number of work pieces indicated by the letter $\mathrm{n}, \mathrm{n}$ can be divided into three stages, in this paper $\mathrm{n}$ is divided into three stages as following: $1<=\mathrm{n}<=7,8<=\mathrm{n}<=20, \mathrm{n}>20$. When $\mathrm{n}$ is the first stage, the simple whole arrangement can be used to find the optimal solution; when $\mathrm{n}$ is the second stage, hybrid heuristic algorithm can be used; when $n$ is the third stage, the limited hybrid heuristic algorithm can be used. The basic idea is as follows:

Step One: Judge the number of work piece, which is the number of columns of the processing time matrix, if the number is less than or equal to 7 , the second step is implemented; if the number is greater than 7 and less than 20 , the third step is implemented; if the number is greater than 20, the sixth step is implemented;

Step Two: Generate and save all possible arrangements of all work pieces, the number of which are the factorial of $n$, then find the make span of all arrangements, and find the minimum make span, output this arrangement;

Step Three: First, put the work piece which has the minimum processing time on the first machine into the first column of scheduling order, and put the work piece which has minimum processing time on the last machine into the second column. Second exchange the arrangement and calculate the make span respectively, put the arrangement which has the smaller make span as optimal arrangement, insert the remaining work pieces into different locations of arrangement in order, seek the minimum make span, save the corresponding arrangement as a new optimal arrangement, and update the arrangement, repeat this process until all work pieces are processed. Finally, calculate make span of all arrangements, find the minimum make span, and output the corresponding arrangement;

Step Four: Obtain the initial arrangement of work pieces based on the Dannerbring method, select the first two work pieces of the arrangement and exchange the order, find the smaller make span and put the corresponding arrangement as a new arrangement, insert the remaining porkpies into different locations of arrangement in order, retain the one with minimum make span, and update the arrangement, until all work pieces are completed. Calculate the make span of all arrangements, to find the minimum make span, and output this arrangement; 
Step Five: Compare the make span of the third step and the fourth step, the smaller one is the final make span and output the corresponding arrangement;

Step Six: First, put the work piece which has the minimum processing time on the first machine into the first column of scheduling order, and put the work piece which has minimum processing time on the last machine into the second column. Second, exchange the arrangement and calculate the make span respectively, put the arrangement which has the smaller make span as optimal arrangement, insert the remaining work pieces into different locations of arrangement in order. Seek the minimum make span, save the corresponding arrangement as a new one. If the number of the new arrangement excess 3 , then take the first ,middle and last one for the new arrangements, and update them until all work pieces are arranged. Calculate the make span of all arrangement, find the minimum make span, and output its arrangement;

Step Seven: Obtain the initial arrangement of work pieces based on the Dannerbring method, select the first two work pieces of the arrangement and exchange the order, find the smaller make span and put the corresponding arrangement as a new arrangement, insert the remaining work pieces into different locations of arrangement in order, retain the one with minimum make span,. If the number of the new arrangement excess 3, then take the first, middle and last one for the new arrangements, and update them until all work pieces are arranged. Calculate the make span of all arrangements, to find the minimum make span, and output its arrangement;

Step Eight: comparing the make span of the step six and the step seven, the smaller one is the final make span and output the corresponding arrangement.

\section{B. Implementation of Flow Shop Problem Solving with Hybrid Constructive Heuristic Algorithm}

Judge the number of rows ('rows' means the number of machines) and columns ('cols' means the number of work pieces) of the input variable - "processing time matrix". According to the value of the cols, the cols is divided into three branches, when $1<=$ cols $<=7$, run the full array solution, when $8<=$ cols $<=30$, run the hybrid heuristic algorithm, when the cols $>30$, the limited hybrid heuristic algorithm can be used.

\section{1) Solution with the full array}

Use the first two columns of the time matrix, swap positions to generate two new arrangements, when the number of $\mathrm{i}$ (means the columns) is from 3 to cols, initialized arrangements are recorded as zero matrix of rows row, i column, $i^{*}$ dimensions (dimension mean the number of new arrangements) dimension, and insert the new column into different locations of arrangement to create new arrangements, until the $\mathrm{i}$ is equal to cols. So there are many new arrangements which number is the factorial of cols. Calculate the make span of each arrangement, and output the minimum make span and its arrangement.

2) Solution with Constructive Hybrid Heuristic Algorithm

\section{a) Heuristic algorithm based on the processing} time

Electing the column of which the processing time is minimal in the first line as the first column, and electing the column of which the processing time is minimal in the rows line as the second column, to generate a new arrangement, when the number of columns(i) is from 3 to cols, initialized arrangements are recorded as zero matrix of rows row, i column, i*dimensions (dimension mean the number of new arrangements) dimension, and insert the new column into different locations of arrangement to create new arrangements, find the minimum make span, and output the all arrangements which meet the make span, update the arrangement, until the $\mathrm{i}$ is equal to cols. Calculate the make span of the arrangements, find the minimum and output the make span and corresponding arrangements.

b) Hybrid Heuristic Algorithm based on the Dannerbring

By the two formulas as following, translate the flow shop problem of multi-machine into two-machine scheduling problem with $\mathrm{Tj} 1, \mathrm{Tj} 2$ as processing time; obtain the initial solution by Johnson method.

$$
\begin{gathered}
T_{j 1}=\sum_{i=1}^{m-1}(m-i+1) * t_{i j} . \\
T_{j 2}=\sum_{i=2}^{m} i * t_{i j} .
\end{gathered}
$$

Calculate the total processing time with the first two columns of the initial solution, take the minimum processing time as the new arrangement, when the number of columns(i) is from 3 to cols, initialized arrangement is recorded as zero matrix of rows row, $i$ column, $i^{*}$ dimensions (dimension mean the number of new arrangements) dimension, and insert the new column into different locations of arrangement to create new arrangements, find the minimum make span, and output the all arrangements which meet the make span, update the arrangements, until the $\mathrm{i}$ is equal to cols. Calculate the make span of the arrangements, find the minimum and output the make span and corresponding arrangements.

3) Limited Hybrid Heuristic Algorithm

As 4.2, the arrangements which meet the minimum make span may be more than one, especially in the first issue of the Hel. Because the processing time is less than 10 , and the number of the work pieces is up to the 100 , the number of same as the best value is a lot, when the number of work pieces increases, the dimensions of the arrangement is in a geometric growth, both computational complexity and computing time will increase a lot, so this paper suggests to use the limited hybrid heuristic algorithm. In each cycle, if there are more than 3 optimal alignments, then retain the first, middle and the last arrangement, update the new arrangement, and the next cycle continues, until all work pieces are arranged, calculate the make span of each arrangement, and output the minimum make span and its arrangements. 


\section{Test of the New Hybrid Constructive Heuristic Algorithm}

This research focused on the test of Car and Hel problems from a typical Flow Shop Scheduling Problem, which is from the book "shop scheduling and its genetic algorithms" written by Wang Ling. And compared the performances of Dannenbring method, Nawaz - Enscore Ham (NEH) method, Rajendran method and the new constructive heuristic method, if there are more than three optimal arrangements, the other methods can also remain the first one, the middle one and the last one. Through this approach, the complexity of each method in calculation is limited, and the time differences on searching for optimization is very small, even we can ignore the differences. The results are shown in Tab. 1 and Tab. 2.

TABLE I. THE CONTRAST OF MAKESPAN BY DIFFERENT METHODS

\begin{tabular}{|c|c|c|c|c|c|c|c|c|}
\hline \multirow{2}{*}{\multicolumn{2}{|c|}{$\begin{array}{c}\text { Typical } \\
\text { problems }\end{array}$}} & \multirow{3}{*}{$\begin{array}{l}\mathbf{n} / \mathbf{m} \\
11 / 5\end{array}$} & \multirow{3}{*}{\begin{tabular}{|c|}
$\begin{array}{c}\text { Make span } \\
\text { standard }\end{array}$ \\
7038 \\
\end{tabular}} & \multirow{3}{*}{\begin{tabular}{|c} 
Dannen \\
-bring
\end{tabular}} & \multirow{3}{*}{$\begin{array}{l}\mathbf{N e H} \\
7038\end{array}$} & \multirow{3}{*}{$\begin{array}{c}\begin{array}{c}\text { Raje } \\
\text {-ndra }\end{array} \\
7038\end{array}$} & \multicolumn{2}{|c|}{ My method } \\
\hline & & & & & & & \multirow{2}{*}{$\begin{array}{c}\text { Sum* } \\
7038\end{array}$} & \multirow{2}{*}{\begin{tabular}{|r|} 
Num \\
105
\end{tabular}} \\
\hline \multirow{8}{*}{$\begin{array}{c}\text { Car } \\
\text { clas } \\
\text { s }\end{array}$} & Car 1 & & & & & & & \\
\hline & Car 2 & $13 / 4$ & 7166 & 7509 & 7376 & 7391 & 7166 & 369 \\
\hline & Car 3 & $12 / 5$ & 7312 & 7339 & 7483 & 7400 & 7399 & 1 \\
\hline & Car 4 & $14 / 4$ & 8003 & 8357 & 8155 & 8115 & 8003 & 28 \\
\hline & Car 5 & $10 / 8$ & 7702 & 8940 & 8047 & 7779 & 7808 & 1 \\
\hline & Car 6 & $8 / 9$ & 8313 & 9179 & 8813 & 8871 & 8739 & 1 \\
\hline & Car 7 & $7 / 7$ & 6590 & 6760 & 7008 & 7016 & 6590 & 1 \\
\hline & Car 8 & $8 / 8$ & 8264 & 9062 & 8732 & 8821 & 8530 & 2 \\
\hline \multirow{2}{*}{$\begin{array}{c}\text { Hel } \\
\text { clas } \\
\mathrm{S}\end{array}$} & Hel 1 & $100 / 10$ & $510 \sim 513$ & 552 & 518 & 539 & 518 & 3 \\
\hline & Hel 2 & $20 / 10$ & $131 \sim 134$ & 148 & 142 & 145 & 140 & 26 \\
\hline
\end{tabular}

Tab.1 reveals the make spans of the optimal sequence of test problems calculated by Dannenbring method, $\mathrm{NeH}$ method, Rajendran method and hybrid heuristics method. The first column in the table is the problem category, the second column is the number of work pieces ( $n$ indicated the number of work pieces) and the number of machines ( $\mathrm{m}$ indicates that the machine number) of the problems, and the third column is the optimal solutions obtained by other methods or proved in theory, the fourth column is the optimal solution obtained by Dannenbring method, the fifth column is the optimal solution obtained by $\mathrm{NEH}$ method, the sixth column is the optimal solution obtained by Rajendran method, the seventh column is the optimal solution obtained by the new method, the last column is the number of the arrangements which can achieve the minimum make span. The results in Table 1 reveals that the new constructive heuristic method proposed method can improve almost all make spans of the other three constructive methods.

Tab. 2 is the result of Table 1 calculated by the formula 5 , and it indicates the variance between the make spans obtained by these methods and optimality's. The first column in the table is the problem category, second, and the third, fourth, fifth column respectively shows the variance of make span by Dannenbring, $\mathrm{NeH}$, Rajendran, and the proposed methods. As can be seen from Tab. 2, the new method is generally better than Dannenbring, $\mathrm{NEH}$ and Rajendran methods, it can improve the Dannenbring result by $6.2 \%$, and improve the NEH result $4 \%$, and improve Rajendran result by $2.4 \%$.

$$
\operatorname{Dif}_{i j}=\frac{\operatorname{Sum}_{i j}-\operatorname{Sum}_{j}^{*}}{\operatorname{Sum}_{j}^{*}}
$$

$\mathrm{Sum}_{j}^{*}$ denotes the optimality of make span on the No. j problem.

Dif $_{i j}$ denotes the variance of make span of the No. $j$ problem calculated by the No. i method.

TABLE II. THE VARIANCE CONTRAST OF MAKESPAN BY DIFFERENT METHODS

\begin{tabular}{|c|c|c|c|c|c|}
\hline \multicolumn{2}{|c|}{$\begin{array}{c}\text { Typical } \\
\text { problems }\end{array}$} & Dannenbring & $\mathrm{NeH}$ & Rajendra & $\begin{array}{c}\text { My } \\
\text { method }\end{array}$ \\
\hline \multirow{8}{*}{$\begin{array}{l}\text { Car } \\
\text { class }\end{array}$} & Car 1 & 0.111 & 0.000 & 0.000 & 0.000 \\
\hline & Car 2 & 0.048 & 0.029 & 0.031 & 0.000 \\
\hline & Car 3 & 0.004 & 0.023 & 0.012 & 0.012 \\
\hline & Car 4 & 0.044 & 0.019 & 0.014 & 0.000 \\
\hline & Car 5 & 0.161 & 0.045 & 0.010 & 0.014 \\
\hline & Car 6 & 0.104 & 0.060 & 0.067 & 0.051 \\
\hline & Car 7 & 0.031 & 0.069 & 0.070 & 0.000 \\
\hline & Car 8 & 0.097 & 0.057 & 0.067 & 0.032 \\
\hline \multirow{2}{*}{$\begin{array}{c}\text { Hel } \\
\text { class }\end{array}$} & Hel 1 & 0.082 & 0.016 & 0.057 & 0.016 \\
\hline & Hel 2 & 0.130 & 0.084 & 0.107 & 0.069 \\
\hline \multicolumn{2}{|c|}{$\begin{array}{c}\text { Mean } \\
\text { difference }\end{array}$} & 0.081 & 0.040 & 0.044 & 0.019 \\
\hline
\end{tabular}

\section{ApPLICATION OF NEW HEURISTIC ALGORITHM IN IN GENETIC ALGORITHM}

In order to ascertain the improved efficiency of the new heuristic algorithm, we design two simple genetic algorithms. The two algorithms are similar. They have the same processes and the same GA parameters. The only difference is in the production of the initial population. One GA's initial population is optimized by the new heuristic algorithm, and the other whose initial population is randomly generated entirely.

\section{A. Design of GA}

The basic parameters of genetic algorithm are Npop $=$ 40, $\mathrm{Pc}=0.6, \mathrm{Pm}=0.01$ and MaxGen $=80$. The parameters were considered the recommendations of Wang Ling [5] and referred to a group of parameters proposed by De Jong [13] which were later widely used as a standard argument. Based on the parameters, we devise the programs of GA and the specific process can be depicted in Fig.1.

4) Generation of Initial Population 
In order to ensure the validity of the initial population, the new individuals are generated by rearranging the serial numbers of work pieces. Such as the initial processing time, involving four work pieces, the serial number of work pieces is given as [A B C D], then the judgment matrix is $[0,1 / 4,2 / 4,3 / 4,1]$. Every time generating two random numbers which range is $[0,1]$, if the first random number is 0.18 , the corresponding serial number of work piece is $\mathrm{A}$, and if the second random number is 0.56 , the corresponding serial number of work piece corresponding is $\mathrm{C}$, so serial numbers of work pieces in the new individuals is [C B A D]. After repeating this process 40 times which is the size of population, the initial population can be drawn. Although the initial population generated by the process meets the requirements of feasibility, it is debatable on satisfying the requirements of uniformity which means the population covers the most feasible solution space. But considering the purpose of this study is to confirm the improved effect of heuristic algorithms on genetic algorithm, the simple method of generating initial population can be accepted. But in the improvement of genetic algorithm, the niche technology and other technologies are required. If we want the initial population including the result of heuristic algorithm, we can repeat the generating process 39 times, and the last individual of population is the result of heuristic algorithm.

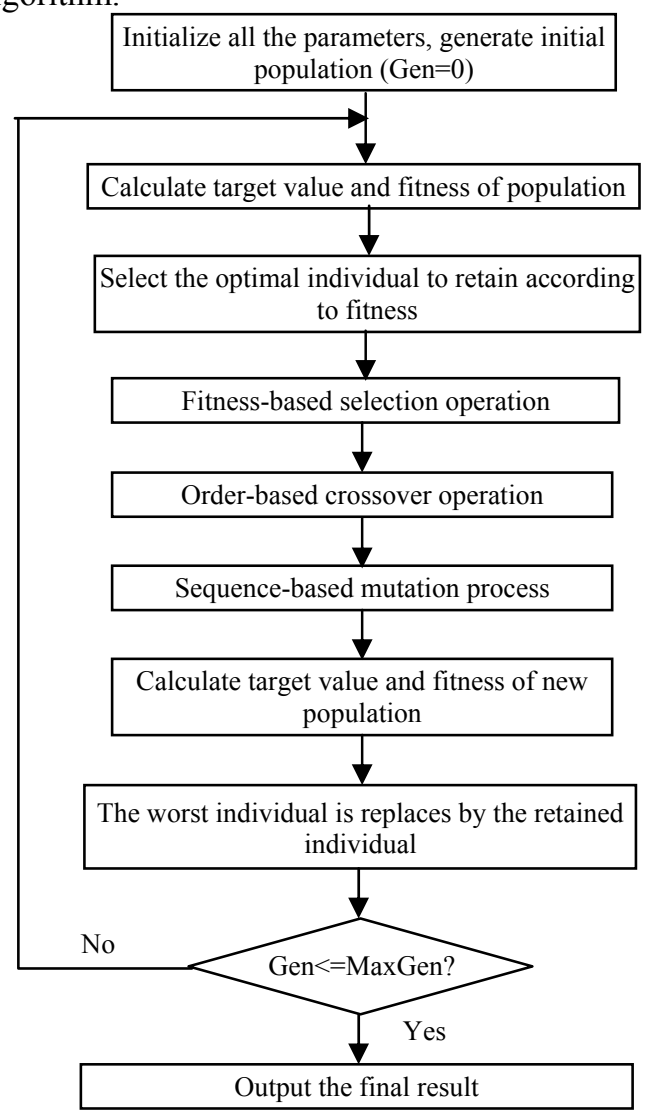

Figure 1. Genetic algorithm flow chart

\section{5) Calculation of the fitness}

The processing time of each individual of population is a two-dimensional array which rows denote machines and columns denote work pieces. When we calculate the make span of scheduling which is the individual of population, we can use the code in Fig. 2. And the "Paixu" in the code denotes the processing time of a scheduling which is a specific arrangement of work pieces. Repeat the program with population size times, the target values of all individuals which are the make spans can be drawn. Due to the make span is better the value is few, it is opposite with the fitness. Therefore, in order to obtain the fitness the conversion formula is needed. There are two conversion formulas, one is the addition formula shown in formula 6 , and the other is multiplication formula shown in formula 7 .

$$
\operatorname{FitnV}_{\mathrm{j}}=\max (\mathrm{Obj} \mathrm{V})-\mathrm{Obj}_{\mathrm{j}}+1 \text {. }
$$

Where: $j=1,2, \ldots \ldots$, Npop

FitnVj denotes the fitness of individuals; $\max (\mathrm{Obj} \mathrm{V})$ denotes the largest processing time;

$\mathrm{Obj} \mathrm{Vj}$ denotes the processing time of $\mathrm{j}$-individuals;

Through the transformation, the target value is smaller, the fitness value is greater, in order to avoid zero value of the fitness, the fitness value of every individual adds one.

$$
\text { Fitn }_{\mathrm{j}}=\max (\mathrm{ObjV}) / \mathrm{Obj}_{\mathrm{j}} \text {. }
$$

Where: $j=1,2, \ldots \ldots$, Npop

Fitn $\mathrm{Vj}$ denotes the fitness of individuals; $\max (\mathrm{Obj} \mathrm{V})$ denotes the largest processing time;

Obj $\mathrm{Vj}$ denotes the processing time of $\mathrm{j}$-individuals;

Because the individual target is Make span, its value is

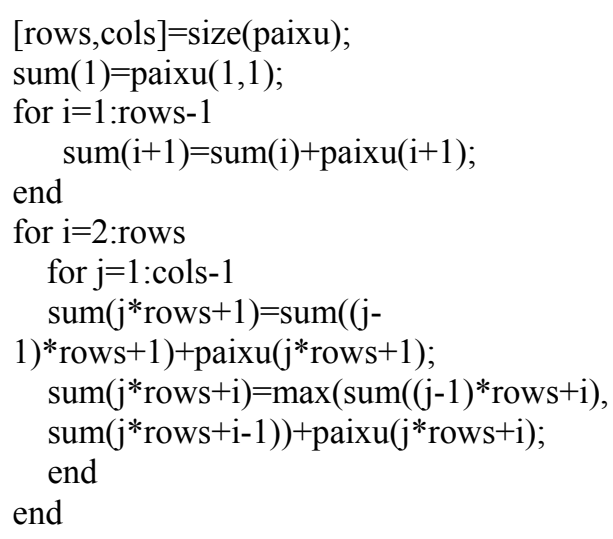

not zero, so the formula is available for all individuals.

Figure 2. The code of calculation of make span

6) Select the retain optimal individual

Sort the population according to fitness, then the highest fitness as the best individual to retain. The detailed code is in Fig. 3.

[FitnV_bl, FitnI_bl]=sort(FitnV); chrom $\overline{\mathrm{N}}=$ chrom(FitnI bl(NIND).: ):

Figure 3. The code of selection the retain individual

\section{7) Fitness-based selection operation}

According to the fitness to generate a judgment which size is one row and Npop columns, and producing a 
random number which range is [0 1], if the comparison judgment, according to the location of judgment which is the random number would fall in to choose the corresponding individual, we can complete the selection operation by the code in Fig. 4.

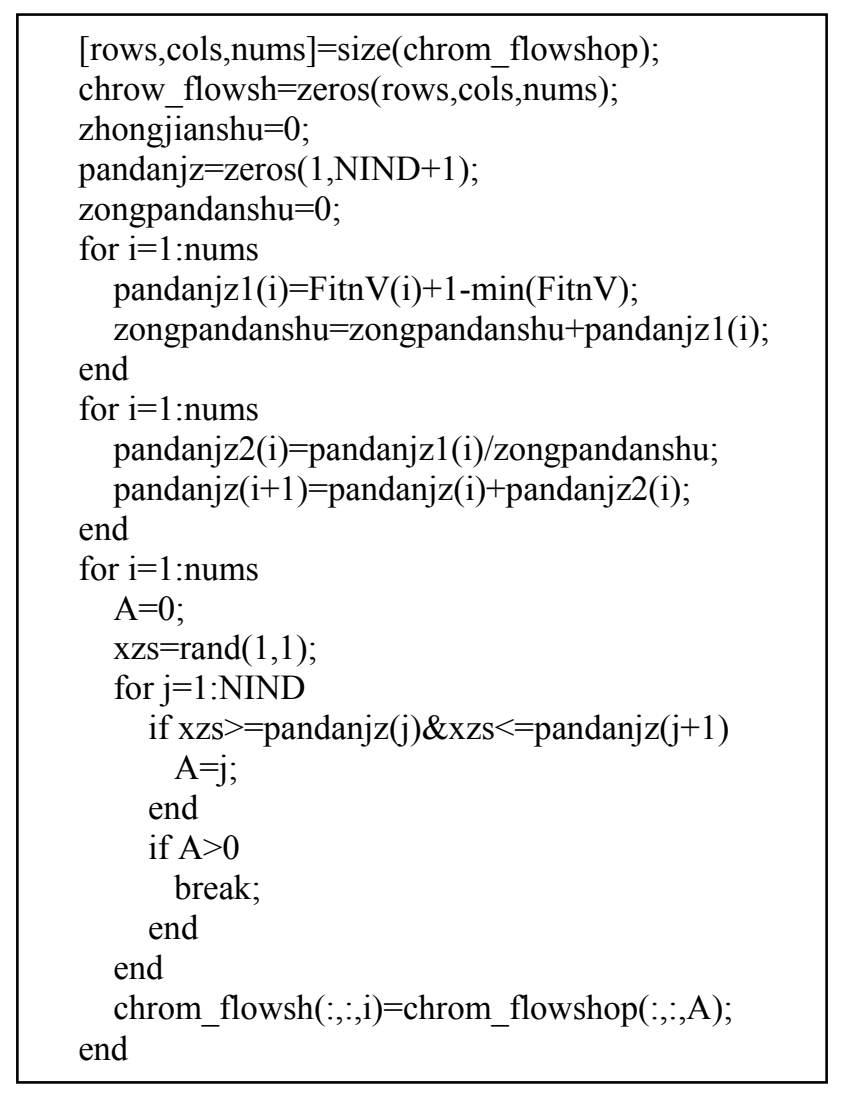

Figure 4. The code of selection operation

\section{8) Order-based crossover operation}

On the basis of the select operation, choosing the first and second individuals, and randomly selecting the cross position, deleting the work pieces of 2 nd individual which is the same as the work pieces of 1 st individual before the cross position, and deleting the work pieces of $1 \mathrm{st}$ individual which is the same as the work pieces of 2nd individual after the cross position, with the rest of work pieces of 2 nd individual to replace the work pieces of the 1st individual after the cross position, the new 1st individual is obtained, and with the rest of work pieces of 1 st individual to replace the work pieces of the 2 nd individual before the cross position, the new 2nd individual is obtained. The method is specifically described in Fig. 5.

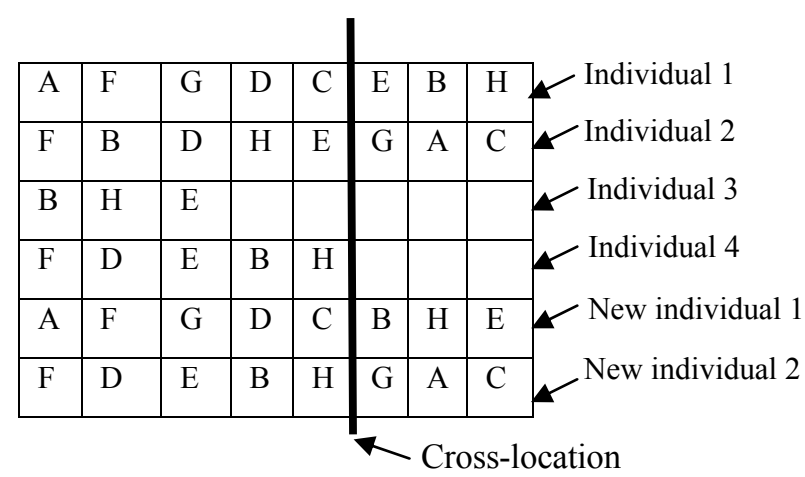

Figure 5. Order-based crossover operation

\section{9) Order-based mutation operation}

In order to ensure the feasibility of solutions, in the mutation operation, we can randomly select two locations; exchange the serial numbers of work pieces on the selected locations. So the mutation operation is completed and the new individual can meet the feasibility requirement. The specific code is in Fig. 6.

Calculating the individual fitness according to the target value of individuals in population is the second step of GA, the second program can be executed directly.

[rows,cols,nums]=size(chrom_gongxu);

jishu_weizhi=zeros $(1,2)$;

pandanjz=zeros $(1,1+$ cols $)$;

for $\mathrm{j}=2: 1+$ cols

pandanjz $(\mathrm{j})=\operatorname{pandanjz}(\mathrm{j}-1)+1 /$ cols;

end

for $\mathrm{i}=1$ :NIND

while jishu_weizhi $(1)==$ jishu_weizhi(2)

jishu_weizhi=rand $(1,2)$;

$$
\begin{aligned}
& \text { for } \mathrm{j}=1: 2 \\
& \quad \text { for } \mathrm{k}=2: 1+\text { cols }
\end{aligned}
$$$$
\text { if }
$$

jishu_weizhi(j) $<=\operatorname{pandanjz(k)\& \& jishu\_ weizhi(j)}>=$ pandanjz(k-1)

$$
\begin{aligned}
& \text { jishu_weizhi }(\mathrm{j})=\mathrm{k}-1 \text {; } \\
& \text { end } \\
& \text { if jishu_weizhi }(\mathrm{j})==\mathrm{k}-1 \\
& \text { break } \\
& \text { end } \\
& \text { end } \\
& \text { end } \\
& \text { end } \\
& \text { if } \mathrm{x} \text { _pandan=rand }(1,1) ;
\end{aligned}
$$

zhongjianshu=chrom_gongxu(:,jishu_weizhi(1),i); chrom_gongxu(:,jishu_weizhi $(1), \mathbf{i})=\overline{c h}$ rom_gongxu (:,jishu_weizhi(2),i);

chrom_gongxu(:,jishu_weizhi(2),i)=zhongjianshu; end end

Figure 6. The code of mutation operation

10) Replace the worst individuals with the best to generate a new population 
After sort the individuals of population according to the fitness, and replace the first individual in the sequence with the best individual, the new population can be gained. The code is in Fig. 7.

$[$ FitnV1,FitnI $]=\operatorname{sort}($ FitnV);

$\operatorname{chrom}(:, ;, \operatorname{FitnI}(1))=$ chromN;

Figure 7. The code of replace operation

Finally, to determine whether the terminating condition is satisfied, if satisfied output the final result, if fail to satisfy go back to the second step repeated the GA until the terminating condition is satisfied.

\section{B. Test of Car problems}

We independently run the above two genetic algorithms 20 times to solve the Car kind problems from Car1 to Car8, and record the results of each run. The results show in Tab. 3, Tab. 4, Tab. 5 and Tab. 6.

Form the results we can see the GA whose initial population is improved by heuristic algorithm obtained more stable satisfactory solution and cost less genetic times, and without using the results of the heuristic algorithm, the solution quality is poor, and cost longer time. Since then, we can see that the proposed heuristic algorithm can effectively improve the result quality of intelligence algorithm.

TABLE III.

COMPARISONS OF TWO GA ON CAR1 AND CAR2

\begin{tabular}{|c|c|c|c|c|c|c|c|c|}
\hline \multirow{3}{*}{ run } & \multicolumn{4}{|c|}{ Car1 } & \multicolumn{4}{|c|}{ Car1 } \\
\hline & \multicolumn{2}{|c|}{ Sim GA } & \multicolumn{2}{|c|}{ Imp GA } & \multicolumn{2}{|c|}{ Sim GA } & \multicolumn{2}{|c|}{ Imp GA } \\
\hline & Opt & gen & Opt & gen & Opt & gen & Opt & gen \\
\hline 1 & 7523 & 62 & 7038 & 1 & 8458 & 1 & 7166 & 1 \\
\hline 2 & 7138 & 30 & 7038 & 1 & 8157 & 51 & 7166 & 1 \\
\hline 3 & 7168 & 25 & 7038 & 1 & 7916 & 54 & 7166 & 1 \\
\hline 4 & 7689 & 9 & 7038 & 1 & 7870 & 80 & 7166 & 1 \\
\hline 5 & 7648 & 27 & 7038 & 1 & 7617 & 62 & 7166 & 1 \\
\hline 6 & 7038 & 54 & 7038 & 1 & 7617 & 50 & 7166 & 1 \\
\hline 7 & 7626 & 70 & 7038 & 1 & 8021 & 74 & 7166 & 1 \\
\hline 8 & 7048 & 11 & 7038 & 1 & 7617 & 56 & 7166 & 1 \\
\hline 9 & 7689 & 9 & 7038 & 1 & 7820 & 56 & 7166 & 1 \\
\hline 10 & 7048 & 57 & 7038 & 1 & 8166 & 3 & 7166 & 1 \\
\hline 11 & 7259 & 38 & 7038 & 1 & 7617 & 70 & 7166 & 1 \\
\hline 12 & 7685 & 58 & 7038 & 1 & 8215 & 5 & 7166 & 1 \\
\hline 13 & 7685 & 21 & 7038 & 1 & 8202 & 7 & 7166 & 1 \\
\hline 14 & 7190 & 69 & 7038 & 1 & 8166 & 9 & 7166 & 1 \\
\hline 15 & 7038 & 21 & 7038 & 1 & 7730 & 54 & 7166 & 1 \\
\hline 16 & 7692 & 61 & 7038 & 1 & 8166 & 5 & 7166 & 1 \\
\hline 17 & 7685 & 18 & 7038 & 1 & 7973 & 15 & 7166 & 1 \\
\hline 18 & 7545 & 77 & 7038 & 1 & 7617 & 48 & 7166 & 1 \\
\hline 19 & 7648 & 21 & 7038 & 1 & 8157 & 26 & 7166 & 1 \\
\hline 20 & 7689 & 8 & 7038 & 1 & 8166 & 3 & 7166 & 1 \\
\hline
\end{tabular}

\begin{tabular}{|l|l|l|l|l|l|l|l|l|} 
Ave & 7436.55 & 37.3 & 7038 & 1 & 7963.4 & 36.45 & 7166 & 1 \\
\hline Opt & 7038 & 54 & 7038 & 1 & 7617 & 48 & 7166 & 1 \\
\hline
\end{tabular}

TABLE IV. COMPARISONS OF TWO GA ON CAR3 AND CAR4

\begin{tabular}{|l|c|c|c|c|c|c|c|c|}
\hline \multirow{2}{*}{ run } & \multicolumn{5}{|c|}{ Car3 } & \multicolumn{2}{|c|}{ Sim GA } & \multicolumn{2}{|c|}{ Imp GA } & \multicolumn{2}{|c|}{ Sim GA } & \multicolumn{2}{|c|}{ Imp GA } \\
\cline { 2 - 9 } & Opt & gen & Opt & gen & Opt & gen & Opt & gen \\
\hline $\mathbf{1}$ & 7710 & 15 & 7399 & 1 & 8479 & 38 & 8003 & 1 \\
\hline $\mathbf{2}$ & 7531 & 57 & 7399 & 1 & 8714 & 74 & 8003 & 1 \\
\hline $\mathbf{3}$ & 7919 & 62 & 7399 & 1 & 8564 & 78 & 8003 & 1 \\
\hline $\mathbf{4}$ & 8171 & 45 & 7399 & 1 & 8426 & 54 & 8003 & 1 \\
\hline $\mathbf{5}$ & 8126 & 18 & 7399 & 1 & 8423 & 61 & 8003 & 1 \\
\hline $\mathbf{6}$ & 7543 & 62 & 7399 & 1 & 9487 & 1 & 8003 & 1 \\
\hline $\mathbf{7}$ & 7594 & 5 & 7399 & 1 & 9487 & 2 & 8003 & 1 \\
\hline $\mathbf{8}$ & 7770 & 27 & 7399 & 1 & 9487 & 33 & 8003 & 1 \\
\hline $\mathbf{9}$ & 7543 & 51 & 7399 & 1 & 9487 & 5 & 8003 & 1 \\
\hline $\mathbf{1 0}$ & 8590 & 5 & 7399 & 1 & 8611 & 64 & 8003 & 1 \\
\hline $\mathbf{1 1}$ & 8567 & 24 & 7399 & 1 & 9487 & 7 & 8003 & 1 \\
\hline $\mathbf{1 2}$ & 7981 & 50 & 7399 & 1 & 9487 & 4 & 8003 & 1 \\
\hline $\mathbf{1 3}$ & 7800 & 59 & 7399 & 1 & 9487 & 3 & 8003 & 1 \\
\hline $\mathbf{1 4}$ & 8255 & 80 & 7399 & 1 & 9487 & 2 & 8003 & 1 \\
\hline $\mathbf{1 5}$ & 8682 & 8 & 7399 & 1 & 9487 & 3 & 8003 & 1 \\
\hline $\mathbf{1 6}$ & 8380 & 16 & 7399 & 1 & 8423 & 73 & 8003 & 1 \\
\hline $\mathbf{1 7}$ & 8099 & 67 & 7399 & 1 & 8611 & 23 & 8003 & 1 \\
\hline $\mathbf{1 8}$ & 7954 & 80 & 7399 & 1 & 9487 & 5 & 8003 & 1 \\
\hline $\mathbf{1 9}$ & 7741 & 76 & 7399 & 1 & 8917 & 58 & 8003 & 1 \\
\hline $\mathbf{2 0}$ & 8126 & 22 & 7399 & 1 & 9487 & 1 & 8003 & 1 \\
\hline Ave & 8004.1 & 41.45 & 7399 & 1 & 9076.25 & 29.45 & 8003 & 1 \\
\hline $\mathbf{O p t}$ & 7531 & 57 & 7399 & 1 & 8423 & 61 & 8003 & 1 \\
\hline
\end{tabular}

TABLE V. COMPARISONS OF TWO GA ON CAR5 AND CAR6

\begin{tabular}{|l|c|c|c|c|c|c|c|c|}
\hline \multirow{2}{*}{ run } & \multicolumn{4}{|c|}{ Car5 } & \multicolumn{4}{c|}{ Car6 } \\
\cline { 2 - 9 } & \multicolumn{2}{|c|}{ Sim GA } & \multicolumn{2}{|c|}{ Imp GA } & \multicolumn{2}{c|}{ Sim GA } & \multicolumn{2}{c|}{ Imp GA } \\
\cline { 2 - 9 } Opt & gen & Opt & gen & Opt & gen & Opt & gen \\
\hline $\mathbf{1}$ & 8047 & 44 & 7720 & 42 & 9507 & 13 & 8570 & 73 \\
\hline $\mathbf{2}$ & 7843 & 79 & 7720 & 7 & 8754 & 11 & 8715 & 25 \\
\hline $\mathbf{3}$ & 8235 & 35 & 7808 & 1 & 9126 & 38 & 8739 & 1 \\
\hline $\mathbf{4}$ & 7862 & 45 & 7750 & 69 & 9355 & 55 & 8715 & 62 \\
\hline $\mathbf{5}$ & 7867 & 62 & 7720 & 24 & 8754 & 58 & 8715 & 36 \\
\hline $\mathbf{6}$ & 7845 & 17 & 7768 & 17 & 9170 & 16 & 8739 & 1 \\
\hline $\mathbf{7}$ & 7862 & 10 & 7750 & 16 & 9170 & 49 & 8739 & 1 \\
\hline $\mathbf{8}$ & 7825 & 8 & 7750 & 69 & 9404 & 10 & 8739 & 1 \\
\hline $\mathbf{9}$ & 7761 & 68 & 7720 & 12 & 9170 & 70 & 8715 & 47 \\
\hline $\mathbf{1 0}$ & 7865 & 9 & 7779 & 45 & 8852 & 60 & 8739 & 1 \\
\hline $\mathbf{1 1}$ & 8518 & 51 & 7750 & 30 & 9267 & 26 & 8715 & 3 \\
\hline
\end{tabular}




\begin{tabular}{|l|c|c|c|c|c|c|c|c|}
$\mathbf{1 2}$ & 7835 & 58 & 7768 & 8 & 8813 & 37 & 8739 & 1 \\
\hline $\mathbf{1 3}$ & 8057 & 76 & 7808 & 1 & 8754 & 49 & 8570 & 3 \\
\hline $\mathbf{1 4}$ & 7822 & 62 & 7750 & 5 & 9293 & 6 & 8570 & 76 \\
\hline $\mathbf{1 5}$ & 7821 & 70 & 7720 & 13 & 9599 & 56 & 8570 & 13 \\
\hline $\mathbf{1 6}$ & 8003 & 10 & 7750 & 32 & 9187 & 14 & 8715 & 7 \\
\hline $\mathbf{1 7}$ & 7867 & 27 & 7808 & 1 & 8715 & 12 & 8715 & 40 \\
\hline $\mathbf{1 8}$ & 7865 & 6 & 7808 & 1 & 8742 & 59 & 8739 & 1 \\
\hline $\mathbf{1 9}$ & 8235 & 13 & 7750 & 59 & 9450 & 72 & 8739 & 1 \\
\hline $\mathbf{2 0}$ & 8039 & 11 & 7720 & 58 & 9179 & 33 & 8715 & 3 \\
\hline Ave & 7953.7 & $/$ & 7755.85 & $/$ & 9113.05 & $/$ & 8695.6 & $/$ \\
\hline Opt & 7761 & 68 & 7720 & 7 & 8715 & 12 & 8570 & 13 \\
\hline
\end{tabular}

TABLE VI. COMPARISONS OF TWO GA ON CAR7 AND CAR8

\begin{tabular}{|l|c|c|c|c|c|c|c|c|}
\hline \multirow{2}{*}{ run } & \multicolumn{5}{|c|}{ Car7 } & \multicolumn{2}{|c|}{ Sim GA } & \multicolumn{2}{|c|}{ Imp GA } & \multicolumn{3}{|c|}{ Sim GA } & \multicolumn{2}{|c|}{ Imp GA } \\
\cline { 2 - 9 } & Opt & gen & Opt & gen & Opt & gen & Opt & gen \\
\hline $\mathbf{1}$ & 6685 & 48 & 6590 & 1 & 9014 & 15 & 8487 & 6 \\
\hline $\mathbf{2}$ & 6779 & 24 & 6590 & 1 & 9091 & 8 & 8530 & 1 \\
\hline $\mathbf{3}$ & 6887 & 19 & 6590 & 1 & 9265 & 59 & 8530 & 1 \\
\hline $\mathbf{4}$ & 6779 & 21 & 6590 & 1 & 8964 & 13 & 8479 & 3 \\
\hline $\mathbf{5}$ & 6803 & 77 & 6590 & 1 & 9313 & 49 & 8409 & 34 \\
\hline $\mathbf{6}$ & 6753 & 30 & 6590 & 1 & 9170 & 60 & 8530 & 1 \\
\hline $\mathbf{7}$ & 6887 & 13 & 6590 & 1 & 9170 & 41 & 8530 & 1 \\
\hline $\mathbf{8}$ & 7037 & 68 & 6590 & 1 & 9365 & 62 & 8530 & 1 \\
\hline $\mathbf{9}$ & 7084 & 54 & 6590 & 1 & 8505 & 71 & 8530 & 1 \\
\hline $\mathbf{1 0}$ & 6590 & 8 & 6590 & 1 & 9267 & 39 & 8479 & 2 \\
\hline $\mathbf{1 1}$ & 6753 & 12 & 6590 & 1 & 9504 & 5 & 8530 & 1 \\
\hline $\mathbf{1 2}$ & 6681 & 33 & 6590 & 1 & 9459 & 77 & 8366 & 22 \\
\hline $\mathbf{1 3}$ & 6753 & 16 & 6590 & 1 & 9068 & 20 & 8366 & 53 \\
\hline $\mathbf{1 4}$ & 6888 & 80 & 6590 & 1 & 9307 & 28 & 8530 & 1 \\
\hline $\mathbf{1 5}$ & 6983 & 7 & 6590 & 1 & 9170 & 49 & 8530 & 1 \\
\hline $\mathbf{1 6}$ & 6760 & 34 & 6590 & 1 & 8871 & 14 & 8530 & 1 \\
\hline $\mathbf{1 7}$ & 6753 & 10 & 6590 & 1 & 9170 & 22 & 8530 & 1 \\
\hline $\mathbf{1 8}$ & 6681 & 26 & 6590 & 1 & 9188 & 13 & 8530 & 1 \\
\hline $\mathbf{1 9}$ & 6753 & 38 & 6590 & 1 & 9226 & 15 & 8473 & 5 \\
\hline $\mathbf{2 0}$ & 6753 & 55 & 6590 & 1 & 8990 & 15 & 8530 & 1 \\
\hline Ave & 6802.1 & 33.65 & 6590 & 1 & 9153.85 & 33.75 & 8497.45 & 6.9 \\
\hline Opt & 6590 & 8 & 6590 & 1 & 8505 & 71 & 8366 & 22 \\
\hline
\end{tabular}

\section{CONCLUSION}

Based on the number of work pieces, a new mixed constructive heuristic algorithm has been designed, and implemented through MATLAB. And contrasting with Dannenbring method, NEH method and Rajendran method by the problems of Car and Hel, the results prove that the new method is better than the other three methods, and it can effectively improve the make span. It can improve the Dannenbring result by $6.2 \%$, and improve the NEH result $4 \%$, and improve Rajendran result by $2.4 \%$. And we discuss the application of the heuristic algorithm in GA. After designing two GAs, one is the simple GA, and the other is improved GA, we test the effect which the heuristic algorithm on GA by eight Car class problems. The result shows using the heuristic algorithm can improve the quality and speed of GA. It can improve the average value by 398.55 and average genetic times by 36.3 on Car. Although it can't improve the optimal value on Car1, it can get the optimal solution with few times by 36.3. And the specific improvement on Car1-8 is in Tab.7 and Tab.8.

TABLE VII. IMPROVEMENT ON VALUES AND GENETIC TIMES ON CAR 1-4

\begin{tabular}{|l|l|r|r|r|l|l|l|r|}
\hline \multirow{2}{*}{} & \multicolumn{2}{|c|}{ Car1 } & \multicolumn{2}{c|}{ Car2 } & \multicolumn{2}{c|}{ Car3 } & \multicolumn{2}{c|}{ Car4 } \\
\cline { 2 - 9 } & Val & \multicolumn{1}{c|}{ Gen } & Val & Gen & Val & Gen & Val & Gen \\
\hline Ave & 398.55 & 36.3 & 797.4 & 35.45 & 605.1 & 40.45 & 1073.25 & 28.45 \\
\hline Opt & 0 & 53 & 451 & 47 & 132 & 56 & 420 & 60 \\
\hline
\end{tabular}

TABLE VIII. IMPROVEMENT ON VALUES AND GENETIC TIMES ON CAR5-8

\begin{tabular}{|l|r|r|r|r|r|r|r|r|}
\hline \multirow{4}{*}{} & \multicolumn{2}{|c|}{ Car5 } & \multicolumn{2}{c|}{ Car6 } & \multicolumn{2}{c|}{ Car7 } & \multicolumn{2}{c|}{ Car8 } \\
\cline { 2 - 9 } & \multicolumn{1}{|c|}{ Val } & Gen & Val & Gen & Val & Gen & Val & Gen \\
\hline Ave & 197.85 & 12.55 & 417.45 & 17.4 & 212.1 & 32.65 & 656.4 & 26.85 \\
\hline Opt & 41 & 61 & 145 & -1 & 0 & 7 & 139 & 49 \\
\hline
\end{tabular}

\section{ACKNOWLEDGMENT}

This paper was supported by National Natural Science Foundation of China under grant number 50875101 and Open Research Foundation of Green Manufacturing and Energy Conservation Technology Research Center under grant number $\mathrm{C} 1010$.

\section{REFERENCES}

[1] M. R. Garey and D. S. Johnson, Computers and Intractability: A Guide to the Theory of NP-Completeness, W.H. Freeman and Company, San Francisco 1979.

[2] K. R. Baker, Introduction to Sequencing and Scheduling, Wiley, New York, 1974.

[3] M. Nawaz, E. Enscore Jr and I. Ham, "A heuristic algorithm for the m-machine, n-job flow-shop sequencing problem," Omega, 11(1), pp. 91-95, 1983.

[4] C. Koulamas, "A new constructive heuristic for the flowshop scheduling problem," European Journal of Operational Research, 105, pp. 66-71, 1998.

[5] Wang Ling, Shop Scheduling with Genetic Algorithms(in chinese), Tsinghua University Press,2003.

[6] F. A. Ogbu and D. K. Smith, "The application of the simulated annealing algorithm to the solution of the $\mathrm{n} / \mathrm{m} /$ Cmax flowshop problem," Computers and Operations Research, 17(3), pp. 243-253, 1990.

[7] C. R. Reeves and T. Yamada, "Genetic algorithms, path relinking and the flowshop sequencing problem," Evolutionary Computation, 6, pp. 45-60, 1998. 
[8] J. Grabowski and J. Pempera, "New block properties for the permutation flowshop problem with application in tabu search," Journal of Operational Research Society, 52, pp. 210-220, 2001.

[9] J. H. Holland, Adaptation in Nartural and Artifical System, MITPress, Massachusett 1975.

[10] D E.Goldberg, Genetic algorithms in search optimization and machine learning reading, Addison Wesley, MA 1989.

[11] L. Wang and D.-Z. Zheng, An Effective Hybrid Heuristic for Flow Shop Scheduling, Int J Adv Manuf Technol (2003) 21:38-44.

[12] Ceyda $\mathrm{OG}^{\smile} \mathrm{UZ}$ and M. Fikert Ercan, A genetic algorithm for hybrid flow-shop scheduling with multiprocessor tasks, Journal of Scheduling 2005, 8, 323-351.
[13] K. A. De JONG, An analysis of the behavior of a class of genetic adaptive systems, University of Michigan, Michigan 1975.

Fang Wang, is female, was born in 1979 and in Hunan province, PR China, and is a PhD student of Huazhong University of Science and Technology (HUST) and a Lecturer of Wuhan University of Science and Technology (WUST), major in Production Scheduling Management.

Yun-qing Rao, is male, was born in 1968 and in Hubei province, PR China, and is a Professor and Mentor, Dr. of Huazhong University of Science and Technology (HUST), major in Production Scheduling and Control.

Yu Hou, is male, was born in 1975 and in Shanxi province, PR China, and is an Associate Professor and Master Instructor of Wuhan University of Science and Technology (WUST), major in Machine Automation. 